\title{
Conservación de la oveja Xalda de Asturias
}

\author{
A. Álvarez Sevilla ${ }^{1}$ J.P. Gutiérrez', I. Fernández ${ }^{3}$, \\ L.J Royo ${ }^{3}$, I. Álvarez ${ }^{3}$ E. Gómez $z^{3}$ \& F. Goyache ${ }^{3}$ \\ ${ }^{1}$ ACOXA, Apdo. de Correos 2117, 33080, Uviéu/Oviedo, Spain \\ ${ }^{2}$ Departamento de Producción Animal, Facultad de Veterinaria de la Universidad Complutense de \\ Madrid, Avda. Complutense s/n, Madrid, Spain \\ 3SERIDA-CENSYRA, C/ Camino de los Claveles 604, 33203 Gijón, Spain
}

\section{Resumen}

En este trabajo se describen las iniciativas de conservación de la oveja de raza Xalda de Asturias. Es una oveja de pequeño tamaño, y de color predominantemente negro que presenta gran similitud morfológica con otras razas ovinas del Arco Atlántico Europeo como la Ouessant o la Morite. En 1992 se funda la asociación de criadores de la raza (ACOXA) y comienza el funcionamiento del Libro Genealógico. Durante la última década la actividad de ACOXA ha estado centrada en la recuperación de individuos aislados fomentando su cría en raza pura. Se ha realizado un análisis de la información genealógica contenida en el Libro Genealógico detectándose el excesivo uso de algunos reproductores que puede provocar una pérdida de variabilidad genética en la población. A partir de estos resultados parece necesario llevar a cabo estrategias de apareamientos que aseguren el mantenimiento del patrimonio genético de la raza.

\section{Summary}

The paper describes the initiatives of conservation of the Xalda sheep breed of Asturias. This is a small sized sheep. The most frequent colour is black. The breed can be related with others of the European Atlantic Arc such as the Ouessant or the Morite. In 1992 the breeders association (ACOXA) founded the Xalda herdbook.
ACOXA began its activities recovering isolated individuals to ensure purebred matings. The pedigree information of the Xalda herdbook was analysed and the abusive use of some individuals was detected. This could lead to looses of genetic variability. New mating strategies should be implemented to preserve the genetic characteristics of the breed.

Keywords: Conservation, Genetic resources, Inbreeding, Effective size.

\section{Introducción}

La oveja de raza Xalda es una población ovina que hasta tiempos recientes no ha recibido especial atención por parte de técnicos y especialistas. Normalmente no ha sido incluida en las descripciones de las diferentes razas de ganado ovino existentes en España (Sánchez Belda y Sánchez Trujillano, 1979). Sin embargo, la oveja Xalda presenta un importante valor de adaptación al medio y su importancia histórica y cultural en el ámbito del Principado de Asturias ha sido claramente establecida (Alvarez Sevilla, 2001). Como resultado del aumento del sentimiento conservacionista en las últimas décadas del siglo XX, en 1992 se creó la Asociación de Criadores de Oveja Xalda de Asturias (ACOXA). La oveja Xalda se incluyó como raza de protección especial en el Real Decreto 1662/1997, de 7 de noviembre, por el que se actualiza el Catálogo Oficial de 
Razas de Ganado de España. El Libro Genealógico gestionado por ACOXA fue reconocido oficialmente por la administración del Principado de Asturias en 1998.

ACOXA está interesada en la mejora del conocimiento de la raza y en la aplicación de estrategias que permitan la conservación de su patrimonio genético (Goyache et al., 2003). En ese sentido, ha impulsado la puesta a punto de un programa de conservación de la variabilidad genética de la raza mediante técnicas de genética de poblaciones y biología molecular. Este programa ha sido financiado por Instituto Nacional de Investigaciones Agrarias y Alimentarias (INIA) mediante el proyecto RZ01-020. En este trabajo se describe la raza Xalda y se analiza su estructura y diversidad genéticas.

\section{Origen e Historia}

La oveja de raza Xalda es una raza ovina en peligro de extinción cuyos efectivos principales se encontraban, en el último

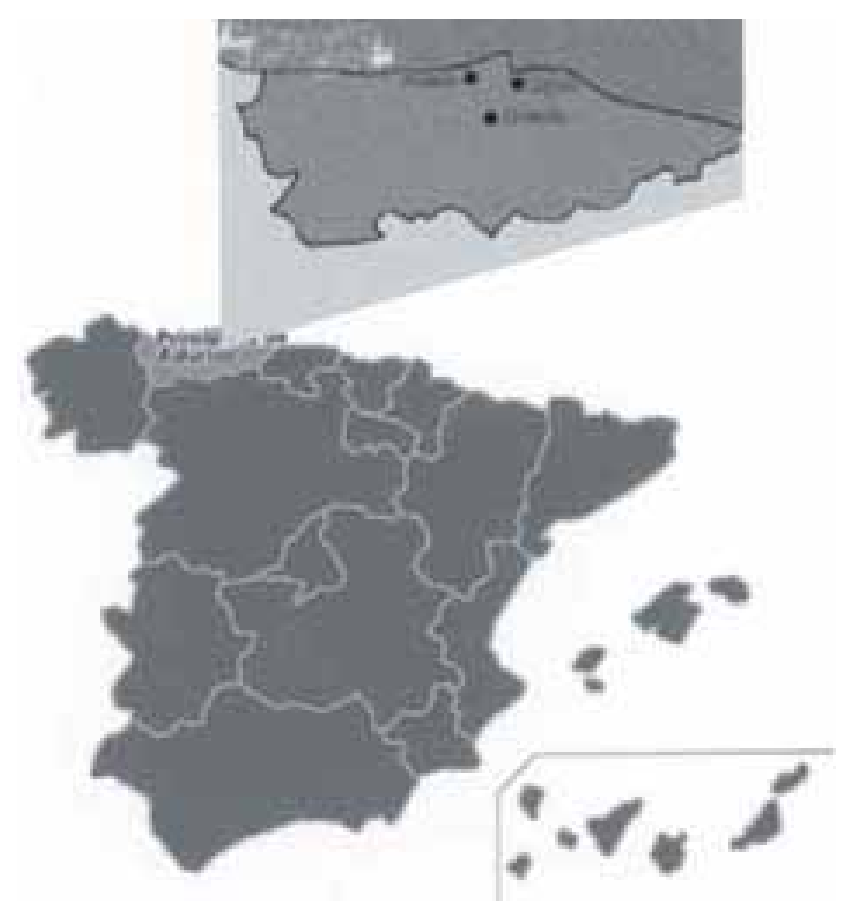

Figura 1. Situación Geográfica de Asturias cuarto del siglo pasado, en el occidente de Asturias, en el norte de España, ligadas a los colectivos humanos de cultura trashumante denominados vaqueiros. La raza fue descrita formalmente por Alvarez Sevilla et al. en 1982 como una población animal propia y diferenciada de las importantes razas ovinas vecinas Churra y Lacha, y que había sido predominante en el vertiente norte de la Cornisa Cantábrica occidental.

Existen fuertes evidencias históricas de la existencia en territorio asturiano de una población ovina que encaja en lo que actualmente conocemos por raza Xalda. Sabemos que los astures poseían ovejas (González, 1976) por dos citas fundamentales: la primera del Edictum Diocletiani de pretiis rerum, 25, en la que se habla de la lana Asturicensis que procedía de Asturias y era considerada como de tercera calidad; la segunda, ésta de Estrabón, al referirse a los astures, nos dice que éstos usaban una túnica o sagun de color negro, al igual que sus capas, pues sus ovejas eran de este color.

A principios del siglo XX la cabaña de raza Xalda era numerosa y tenía gran difusión. Aunque los ganaderos poseían un pequeño número de cabezas, los rebaños de ovejas eran grandes porque la explotación tradicional de esta oveja se realizaba en común mediante la denominada vecera. Existen testimonios gráficos (Krüger, 1949; Uría Ríu, 1976), el primero datado en 1909 (Alvarez Sevilla et al., 1982) que reflejan rebaños de ovejas con las características fenotípicas de las ovejas Xalda en diferentes zonas del centro y el occidente de Asturias. La mayor parte de los animales reflejados en estos testimonios fotográficos son de color negro.

El origen de la raza puede ser el Tronco Ovies aries celticus (Alvarez Sevilla, 1999), muy raro en la Península Ibérica. La raza presenta un fenotipo similar al de otras razas ovinas del arco atlántico como la raza Ouessant de la Bretaña Francesa, la Black Welsh galesa, la Morite de las Islas Shetland de Escocia, la raza Gallega en España y la Bordaleira en el Norte de Portugal. 
La raza comienza su recesión en la segunda mitad del siglo XX por la despoblación de amplias zonas rurales en Asturias y por la agresiva política forestal llevada a cabo por las administraciones públicas que redujeron enormemente los pastos comunales disponibles. La Xalda no resulta una raza competitiva con animales alóctonos en buenas condiciones productivas. La Xalda no es una oveja precoz para la producción de corderos con destino a consumo y la producción de una lana de baja calidad, que era la función principal de estos animales, deja de ser interesante a partir de los años 50. Las ovejas Xaldas no se ordeñan. Alvarez Sevilla et al. (1982) cifraban en no más de 800 hembras reproductoras puras el censo de la raza en 1980.

\section{Características Morfológicas}

La principales características están resumidas en la Tabla 1.

Tabla 1. Características morfológicas de la oveja Xalda de Asturias.

\begin{tabular}{ll}
\hline Cabeza & $\begin{array}{l}\text { Pequeña, perfil siempre recto en las hembras (incluso ligeramente } \\
\text { cóncavo) y subconvexo en los carneros. }\end{array}$ \\
\hline Ojos & Grandes y vivarachos. Cuencas prominentes. \\
\hline Orejas & Pequeñas y dirigidas hacia adelante. \\
\hline Cuello & $\begin{array}{l}\text { De longitud media y firme, más en los machos que en las hembras. } \\
\text { Presentan a lo largo del mismo y hasta el pecho, pelos que le dan cierto } \\
\text { carácter muflónico. Por la parte baja de la nuca, aparece una especie de } \\
\text { crinera, formando una abundante pelambrera. El rabo es corto, llegando } \\
\text { a la altura de los corvejones. }\end{array}$ \\
\hline Tronco & $\begin{array}{l}\text { Alargado. Recto en las hembras, encorvado en la zona de la cruz en el } \\
\text { carnero. Costillar con buena curvatura. }\end{array}$ \\
\hline Extremidades & Finas, con pezuñas pequeñas y duras. \\
\hline Piel & Fina. Sin pliegues. \\
\hline Mamas & Bien formadas. Pezones pequeños. \\
\hline Colornos & $\begin{array}{l}\text { Solo los presentan los machos. Su forma es en espiral envuelta, apretada, } \\
\text { de aspecto característico, tallada por surcos transversales. }\end{array}$ \\
\hline El color puede ser negro, blanco y, en menor proporción, cárdeno. Los \\
animales negros pueden presentar manchas blanca más o menos extensas \\
en la cabeza, hocico y cuello. La lana negra nace muy oscura para \\
aclarase en los extremos hasta tonos caoba cuando es muy larga. El \\
hocico es del mismo color de la capa salvo cuando se ve afectado por una \\
mancha blanca en animales negros.
\end{tabular}




\section{El Libro Genealógico}

El Libro Genealógico de la Oveja de raza

Xalda de Asturias comenzó su funcionamiento oficial en 1992 con la constitución de ACOXA. En él se han incluido las genealogías recogidas por los promotores de ACOXA en los años previos a su fundación. Durante los últimos años de la década de los 80 y principios de los 90, la labor de los socios de ACOXA consistió en identificar ejemplares en rebaños aislados que se han ido incorporando progresivamente al Libro Genealógico. De acuerdo con el origen geográfico de los animales y de su importancia en la conservación de la raza, ACOXA reconoce la existencia de 9 líneas diferenciadas: Adrao, Antón, Arquil.lina, Brañaseca, Candaneu, Eilao, Eirrondo, Ixuxú y Oubal.lu. La mayoría de los moruecos utilizados y sus madres provienen de esas líneas fundacionales. El Libro Genealógico cuenta con un total de 805 animales inscritos de los que 562 están vivos (507 hembras). Los animales registrados sin padres conocidos son 329 (58 machos y 271 hembras). Estos animales se consideran fundadores de la raza. El número de fundadores se eleva a 409 si consideramos los animales de los que sólo conocemos un progenitor. Durante la mayor parte de los años 90 el porcentaje de hembras sin genealogía incorporadas al Libro Genealógico ha superado el 50\% de las hembras totales registradas. La población
Xalda registrada es mayoritariamente joven con un 39\% de los animales menores de dos años y un $45 \%$ de entre 3 y 5 años de edad.

Aunque el número de rebaños que aportan animales cada año al Libro Genealógico llega a un máximo de 26 en el año 2001, el número total de rebaños registrados en el Libro Genealógico es de 62. Esto se debe tanto al interés de ACOXA en registrar el origen de los animales fundacionales como a la poca pervivencia en el tiempo de muchos rebaños, lo que provoca gran movilidad de los animales. La contribución de los moruecos de raza Xalda al Libro Genealógico se puede observar en la Tabla 2. La mayoría de los 50 moruecos utilizados produjeron 6 hijos o menos mientras que 9 moruecos produjeron más de 20 hijos. Sólo se han registrado 30 o más hijos de 5 moruecos. Estos 5 moruecos han contribuido a la raza con el $31 \%$ de los animales registrados con padre conocido. Un $60 \%$ de los rebaños sólo han registrado 10 animales o menos. Los 5 rebaños que han registrado más de 40 animales son responsables del $34 \%$ de los animales incluidos en el Libro Genealógico.

En la Tabla 3 se detallan los rebaños que aparecen representados en mayor porcentaje en el Libro Genealógico de la oveja Xalda mediante la utilización de sus machos como padres, como abuelos y por el número total de apariciones como progenitores de los moruecos producidos en ese rebaño (Barker, 1957). Los rebaños cuyos moruecos aparecen

Tabla 2. Porcentaje de moruecos y rebaños por el número de an imales incluidos en el Libro Genealógico de la raza Xalda.

\begin{tabular}{lccccc}
\hline & \multicolumn{2}{c}{ Moruecos } & & \multicolumn{2}{c}{ Rebaños } \\
\cline { 2 - 3 } \cline { 5 - 6 } & Número & Porcentaje & & Número & Porcentaje \\
\hline Menos de 6 & 24 & 48 & & 18 & 29,0 \\
De 6 a 10 & 6 & 12 & & 18 & 29,0 \\
De 11 a 20 & 11 & 22 & & 9 & 14,6 \\
De 21 a 40 & 9 & 18 & & 7 & 11,3 \\
De 40 a 80 & & & & 3 & 4,8 \\
Mas de 80 & & & & 7 & 11,3 \\
\hline
\end{tabular}




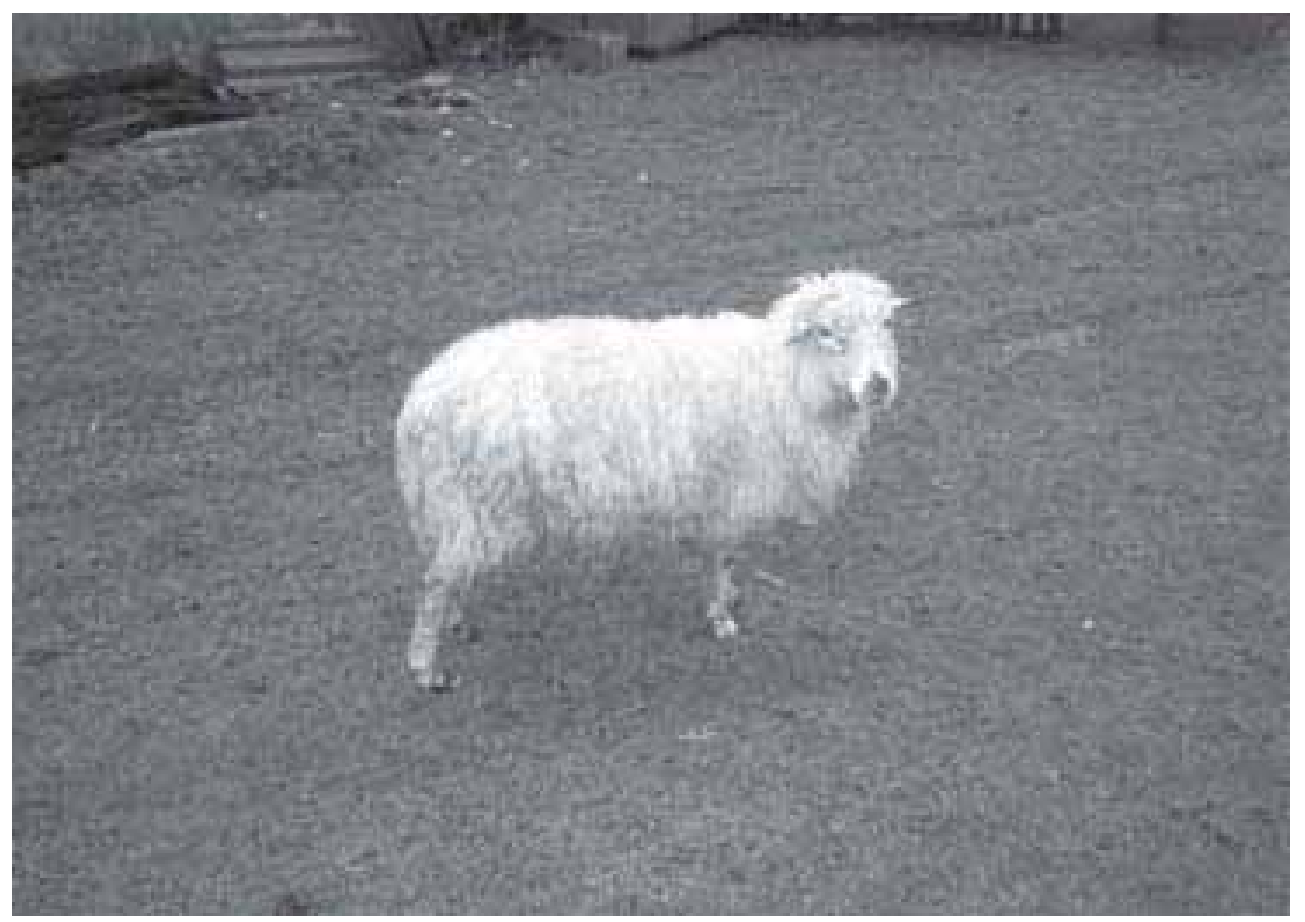

Tabla 3. Rebaños más importantes en el Libro genealógico de la oveja Xalda por número de veces (en porcentaje) que aparecen los moruecos producidos en un rebaño como padre y como abuelos.

\begin{tabular}{lcccc}
\hline Rebaño & Concejo & & Como padres & Como abuelos \\
\cline { 1 - 2 } \cline { 5 - 5 } \cline { 5 - 5 } Aurelio Esteban & Ribera d'Arriba & & 9,6 & 23,92 \\
Antón Alvarez & Llanera & & 14,0 & 20,34 \\
Brañaseca & Cuideiru & & 8,3 & 8,29 \\
Stefan Laurencery & Somiedu & & 6,1 & 9,42 \\
Santiago Díaz & Llanera & & 2,4 & 10,73 \\
Candaneu & Tineu & & 1,7 & 5,65 \\
Marta Vega & Sobrescobiu & & 9,8 & 1,88 \\
Francisco Pañeda & Noreña & & 3,6 & 1,69 \\
Severino García & Villaviciosa & & 5,9 & 5,65 \\
Oubal.lu & Cangas del Narcea & & 4,4 & 5,46 \\
Marino García & Sieru & & 5,6 & 2,07 \\
Cooperativa Xedré & Cangas del Narcea & 5,7 & 0,00 \\
\hline
\end{tabular}

en total, al menos, un $5 \%$ de las veces son 12 (el $18 \%$ del total) y suman el $84,5 \%$ de los sementales utilizados como reproductores en el Libro Genealógico. Sin embargo, sólo 5 de esos rebaños aparecen representados en más de un $5 \%$ como padres y como abuelos, lo que caracteriza la poca persistencia en el tiempo de los rebaños de esta raza.

Las figuras de 2 a 4 muestran respectivamente, una oveja, un carnero y un rebaño de raza Xalda. 


\section{Evaluación de la información genealógica}

La actividad de ACOXA desde su fundación ha estado enfocada a la identificación y recuperación de individuos de la raza promoviendo su utilización en apareamientos en raza pura. Sin embargo, en los últimos años las posibilidades de encontrar nuevos animales en rebaños aislados han disminuido enormemente y en breve plazo la raza Xalda se convertirá en una población cerrada. ACOXA ha promovido el análisis de la información contenida en el Libro Genealógico de la raza para evaluar la variabilidad genética de la raza y de cómo ha podido influir en ella la estrategia de apareamientos seguida hasta ahora (Gutiérrez et al., 2002). Para ello se han evaluado parámetros poblacionales clásicos como el intervalo generacional medio, la evolución de la consanguinidad y el tamaño efectivo de la población (Falconer y McKay, 1996) y, finalmente, el número efectivo de ancestros propuesto por Boichard et al. (1997).

El intervalo generacional es la edad media de los padres a la que sus hijos se convierten, a su vez, en padres y se ha calculado para las cuatro vías (padre hijo, padre hija, madre hijo y madre hija) utilizando las fechas de nacimiento de cada individuo y la de sus padres (Tabla 4). En la raza Xalda la vía padre - hijo es la más corta, puesto que se tiende a conservar un hijo del único semental de que se dispone normalmente. Como

Tabla 4. Intervalos generacionales, en años en la oveja de raza Xalda.

\begin{tabular}{lrl}
\hline Vía padre- hijo & $\mathrm{N}$ & Años \\
Morueco- hijo & 98 & 2,67 \\
Morueco- hija & 434 & 2,86 \\
Oveja - hijo & 91 & 3,23 \\
Oveja - hija & 329 & 3,11 \\
\hline
\end{tabular}

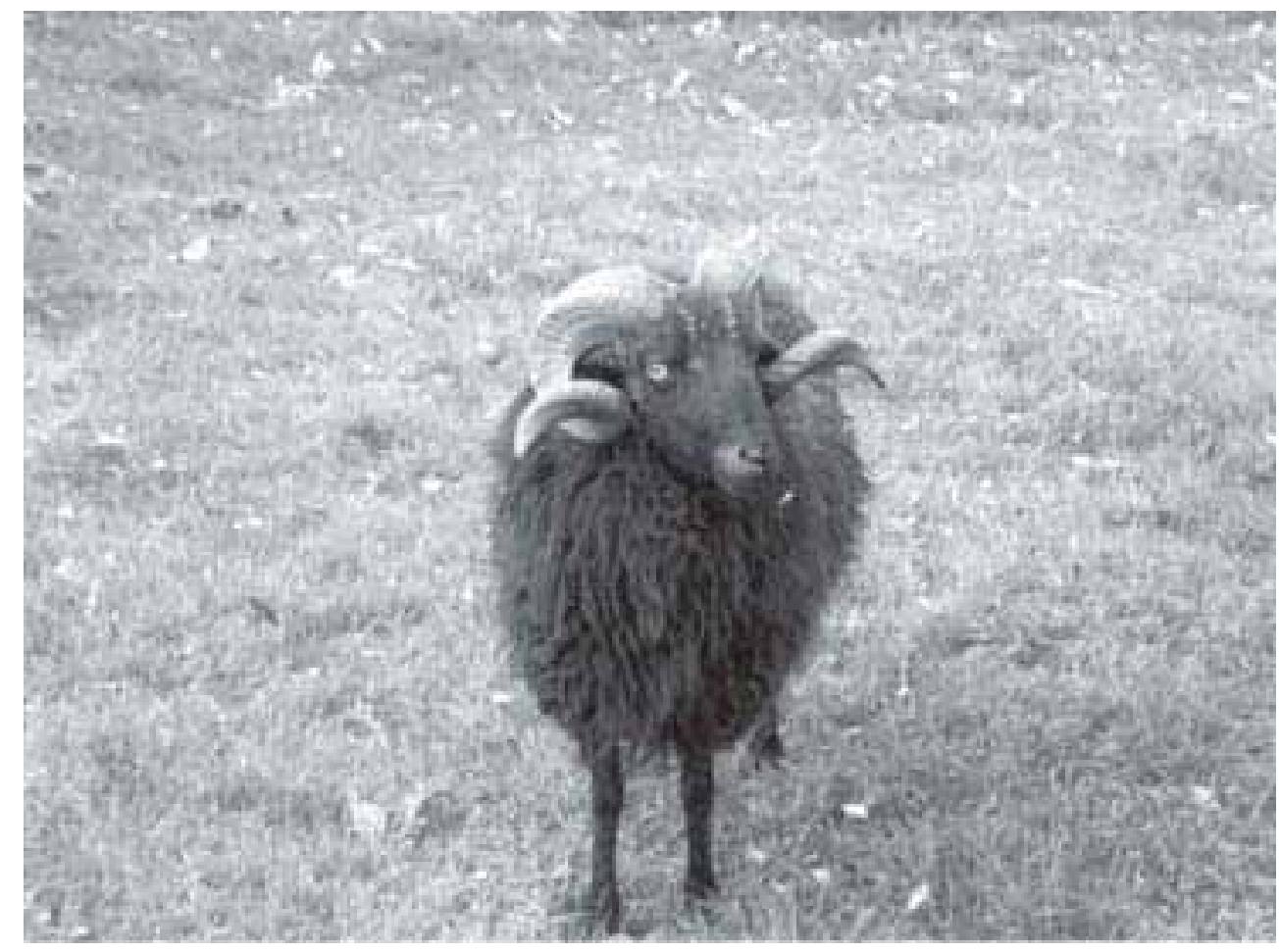

Figura 3. Carnero de raza Xalda. 


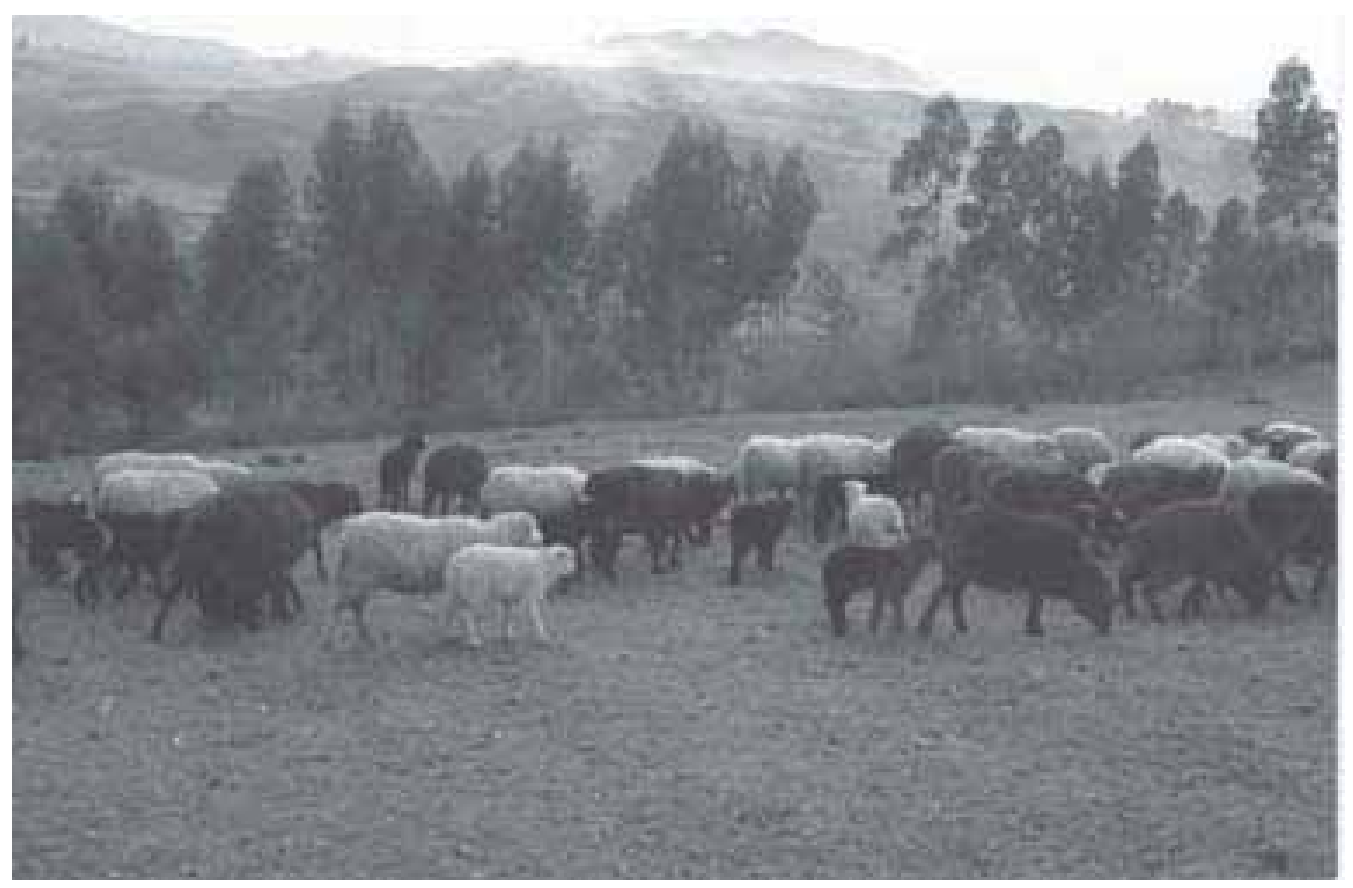

Figura 3. Rebaño de ovejas Xalda.

madre de un futuro semental se utiliza una oveja de la que se tenga suficiente

información (al menos dos partos), por lo que el intervalo generacional madre - hijo es el más largo.

Un parámetro de interés en la descripción de pequeñas poblaciones es el número efectivo de ancestros (Boichard et al., 1997), definido como el número de ascendientes, fundadores o no, necesarios para explicar la variabilidad genética total de la población. Este parámetro tiene en cuenta los posibles cuellos de botella que haya experimentado la población estudiada por una excesiva utilización de determinados reproductores provocando una reducción importante de la variabilidad genética. Este parámetro resulta especialmente informativo sobre la situación de la raza Xalda. El número efectivo de ancestros es de 40 mientras que el tamaño efectivo de la población base calculado con metodologías clásicas (Wright, 1931) es de 81. El tamaño efectivo de la población base estima el número de fundadores necesario para explicar la variabilidad total de la población asumiendo que todos ellos realizan la misma contribución. El número efectivo de ancestros tiene en cuenta que el uso de los reproductores puede estar desequilibrado. El número total de ancestros considerados mediante la técnica de Boichard et al. (1997) es de 236, lo que supone un $29 \%$ de los animales de la población. Estos 236 animales explicarían toda la variabilidad genética de la raza. Un número muy pequeño de ancestros (15) explica rápidamente una gran parte de la variabilidad genética de la población (50\%). Con sólo 36 animales se explica el $70 \%$ de la variabilidad genética de la población. Este hecho parece indicar que la raza se encuentra sometida a cierto grado de selección por parte de los criadores que tienden a utilizar masivamente un reducido número de reproductores que producen hijos con los caracteres de tipo que se consideran más deseables en la raza.

La población Xalda presenta una consanguinidad media de 1,5\%. Los animales endogámicos son aproximadamente el $11 \%$ de la población y su consanguinidad media es muy alta $(14,4 \%)$. La consanguinidad media se incrementa rápidamente con la profundidad del conocimiento del pedigrí, presentando valores que superan la media de la población a partir de la tercera generación conocida 
hasta situarse en un 13\% a partir de la sexta (Tabla 5). La consanguinidad en la raza Xalda se ha acumulado fundamentalmente en los años de 1999 a 2001, años en los que la incorporación de animales sin genealogía conocida al Libro Genealógico ha sido muy pequeña. Aunque Prod'Homme y Lauvergne (1993), en un núcleo cerrado de raza Rambouillet, estiman valores de endogamia de entre el 37 y el $48 \%$ sin encontrar consecuencias en caracteres productivos o reproductivos, los valores medios de endogamia y parentesco medio de la población Xalda aconsejan una cuidadosa planificación de los apareamientos a realizar para evitar una disminución importante de la variabilidad genética. Los moruecos endogámicos son el 10\% de los utilizados con una consanguinidad media del $12,5 \%$, mientras que la endogamia media de los machos activos es del 1,3\% . Estos valores son superiores a los aceptados por Djellali et al. (1994) en los programas de conservación de las razas Solognote y Merino precoz que encuentran un 10\% de machos activos endogámicos pero una endogamia media de los machos activos del 0,5\%.

\section{Conclusiones y Trabajo Futuro}

Alvarez Sevilla et al. (1982) definieron la oveja Xalda como una raza abierta, por carecer de norma selectiva y haber quedado su evolución a exclusiva iniciativa de cada ganadero. Siguiendo a Sánchez Belda y Sánchez Trujillano (1979) podía considerarse como una raza en regresión, amenazada de extinción y en estado de reliquia.

La fundación de ACOXA ha permitido un cambio en la situación de la raza. Se ha recuperado un importante número de reproductores que permite asegurar el mantenimiento de la población. Sin embargo, la variabilidad genética que aportan es limitada, por lo que resulta necesario instrumentar medidas que aseguren la máxima representación genética de los animales fundadores del Libro genealógico en la población presente. En especial parece necesario establecer estrategias de gestión de reproductores que eviten la utilización abusiva de un número limitado de individuos lo que produciría un empobrecimiento del patrimonio genético de la raza.

Además ACOXA está interesada en resaltar los valores distintivos de la raza para promover su conocimiento y aprecio en la sociedad asturiana. Los valores culturales y estéticos de nuestros animales es muy apreciado en establecimientos de turismo rural y ecomuseos. La raza presenta una gran rusticidad que le permite estar perfectamente adaptada al pastoreo en zonas de montaña con clima lluvioso y bruscos cambios de temperatura. Sin embargo, la raza no resulta competitiva con razas mejoradas en condiciones productivas

Tabla 5. Número de animales de raza Xalda y valores medios de consanguinidad y parentesco medio (en porcentajes) por número de genenciones conocidas.

\begin{tabular}{lcc}
\hline Generación & Animales & Consangunidad (\%) \\
\hline 0 (Población Base) & 249 & 0 \\
1 (Un padre conocido) & 105 & 0 \\
2 (Un abuelo conocido) & 188 & 1,4 \\
3 (Un bisabuelo conocido) & 72 & 2,0 \\
4 (Un tatarabuelo conocido) & 145 & 3,3 \\
5 (Un tataratatarabuelo comocido) & 39 & 6,6 \\
$>5$ (Resto) & 7 & 13,0 \\
\hline
\end{tabular}


estándar. La raza Xalda no es precoz y el porcentaje de partos gemelares es de alrededor de un $3 \%$. El cordero comercial de raza Xalda debe tener 7-8 meses de edad. El mantenimiento de las líneas de apoyo económico de las administraciones públicas a los criadores de oveja Xalda iniciado en 1999 resulta esencial para el mantenimiento de la población.

\section{Bibliografía}

Alvarez Sevilla, J.A., García Peláez, A., Corté Pérez, J. 1982. Descripción de la oveja de raza Asturiana. Biol Cien Nat I.D.E.A., 30: 147-157.

Alvarez Sevilla, J.A. 1999. Protohistoria y ganadería. En El Ganado vacuno del Tronco Castaño, pp.11-18, Imprenta Narcea S.L., Granda-Siero (Asturias).

Alvarez Sevilla, A. 2001. Les races autóctones del Principáu d'Asturies, Fundación Belenos, 33007 Uviéu/Oviedo (Prinicpau d'Asturies), 11-18.

Barker, J.S.F. 1957. The breed structure and genetic analysis of the pedigree cattle breeds in Australia. I: The Jersey. Aust. J. Agric. Res. 8, 561-586.

Boichard, D., Maignel, L. \& Verrier, E. 1997. The value of using probabilities of gene origin to easure genetic variability in a population, Genet. Sel. Evol. 29: 5-23.

Djellali, A., Vu Tien Khang, J., de Rocambeau, H. \& Verrier, E., 1994. Bilan génétique des programmes de conservation de races ovines Solognote et Mérinos précoce. Genet. Sel. Evol. 26, Suppl 1, 255s-265s.
Falconer, D.S. \& Mackay, T.F.C. 1996. Introduction to Quantitative Genetics, Longman, Harlow.

González, J. M., 1976. Antiguos Pobladores de Asturias (Protohistoria). Ayalga Ed. Salinas, pp. 212.

Goyache, F., Gutiérrez, J.P., Fernández, I., Gómez, E., Álvarez, I. Díez, J. \& Royo, L.J. 2003. Using pedigree information to monitor genetic variability of endangered populations: the Xalda sheep breed of Asturias as an example. J. Anim. Breed. Genet. 120: 95-103

Gutiérrez, J.P., Fernández, I., Royo, L.J., Álvarez, I., García, G., Álvarez Sevilla, A. \& Goyache, F. 2002. Análisis demográfico y genético de la población ovina de raza Xalda de Asturias. V Congreso de la SERGA. 18-20 de septiembre de 2002.

Krüger, F. 1949. Las Brañas. Bol. I.D.E.A., 8: 41-96.

Prod'Homme, P. \& Lauvergne, J.J. 1993. The Merino Rambouillet flock in the National Sheep Fold in France, Small Rumin. Res. 10: 303-315.

Sánchez Belda, A. \& Sánchez Trujillano, M.C. 1979. Razas Ovinas Españolas. Publicaciones de Extensión Agraria. Madrid, pp. 501.

Uría Ríu, J. 1976. Los Vaqueiros de alzada y otros estudios (de caza y etnografía). Obras Completas. Vol. 1. Biblioteca Popular Asturiana. Oviedo, pp. 346.

Wright S. 1931. Evolution in mendelian populations, Genetics, 16: 97-159. 